\title{
LA GEOLOCALIZACIÓN SOCIAL
}

\author{
Gersón BELTRÁN LÓPEZ (gerson.beltran@gmail.com) \\ Departamento de Geografía. Universitat de València
}

Recibido: 27/03/2015

Aceptado: 14/07/2015

RESUMEN: La geolocalización es una de las herramientas utilizadas por los geógrafos para situar a las personas u objetos en el espacio mediante sus coordenadas y que ha cobrado una nueva dimensión a partir de la aparición de Internet y de los dispositivos móviles. Paralelamente se ha desarrollado el fenómeno de compartir información desde cada lugar e individuo en los social media (medios sociales). A la unión de estos aspectos se le ha denominado SoLoMo, acrónimo de social, local y móvil, donde la geolocalización es una herramienta de comunicación entre el mundo físico y el digital, desde lo local a lo global, desde el lugar a la nube. Por tanto geolocalización y redes sociales son dos elementos complementarios, otorgando una nueva dimensión a la geografía y que deben ser analizados en un contexto de modernidad líquida. En este artículo se realiza una revisión de estos conceptos nuevos en la geografía, pero imprescindibles en el análisis espacial, que hoy en dia no puede entenderse sin Internet.

PALABRAS CLAVE: geolocalización social, geolocalización, geomarketing, social media, solomo, web 2.0., neogeografía, geografía, análisis espacial, internet, big data.

\section{SOCIAL GEOLOCATION}

ABSTRACT: Geolocation is one of the tools used by geographers to place people or objects in space using their coordinates and which has taken on a new dimension since the emergence of the Internet and mobile devices. In parallel, the phenomenon of sharing information from a location by an individual on social media (social networks) has developed. The union of these aspects has been called SoLoMo, an acronym of social, local and mobile, where geolocation is a communication tool between the physical and the digital world, from local to global, from the location to the cloud. Therefore geolocation and social networks are two elements which complement each other, giving geography a new dimension and which must be studied in a context of liquid modernity. This article reviews these concepts which are new to geography, but essential to spatial analysis, which today cannot be comprehended without Internet.

KEY WORDS: social geolocation, geolocation, geomarketing, social media, solomo, web 2.0., neogeography, geography, spatial analysis, internet, big data.

\section{ANTECEDENTES}

Hace ya años Castells habló de la Sociedad Red en su magnífica trilogía donde reflexionaba sobre los cambios que Internet estaba suponiendo e iba a suponer en la sociedad (CASTELLS, 2005) Años después otro sociólogo, Bau- 
man, habló de la sociedad líquida para definir un estado de la sociedad posmoderna en los últimos años donde las tradicionales estructuras sólidas que conformaban la sociedad industrial y postindustrial estaban siendo superadas y habían cambiado a una estructura líquida, flexible, que se adaptaba a las circunstancias con una gran celeridad (BAUMAN, 2003).

La geografía es una ciencia, o al menos se define como tal después de los análisis de Alexander Von Humbolt y ha pasado por muchas etapas. Horacio Capel hizo una revisión de la epistemología geográfica en su libro «Filosofía y ciencia en la Geografía contemporánea. Una introducción a la Geografía» hasta analizar la situación de la ciencia geográfica a finales del siglo pasado (CAPEL, 1981).

Hace unos años el mismo autor, Horacio Capel, volvía a reflexionar y en su artículo titulado «Geografía en red a comienzos del tercer milenio: para una ciencia solidaria y en colaboración» hacía un análisis de los cambios que la aparición de la Internet y la denominada web 2.0. había supuesto en la Geografía y las posibilidades que ello ofrecía al trabajo en red y colaborativo (CAPEL, 2010).

Este artículo parte de la hipótesis de que la sociedad ha cambiado hacia un estado líquido, mientras que algunas ciencias como la geografía se mantienen en postulados industriales, incapaz de dar respuesta a muchos de los cambios de esta nueva era.

Es necesario pues realizar un ejercicio de reflexión al respecto y, al mismo tiempo, introducir en la investigación geográfica la aparición de una neogeografía o geografía 2.0. La geografía no puede permitirse hablar de la sociedad desde las sillas de los despachos como decía el geógrafo del Principito «El geógrafo no puede estar de acá para allá contando las ciudades, los ríos, las montañas, los océanos y los desiertos; es demasiado importante para deambular por ahí. Se queda en su despacho y allí recibe a los exploradores» (De SAINT-EXUPÉRY, 1998), aunque se da la paradoja de que en estos momentos se puede «salir» virtualmente del despacho sin moverse de la silla a través de Internet.

La geografía es una ciencia al servicio de la sociedad, es un medio para lograr un fin, que es la mejora de la sociedad y no al revés, un fin al que la sociedad debe adaptarse. Por tanto hay que escuchar a la sociedad, adaptarse a sus necesidades y voluntades y para ello hay que descender al nivel de la calle, la geografía en estos momentos vuelve a tener un componente subjetivo que recuerda al paradigma behavioruista de los años setenta, la geografía subjetiva (BELLOVIN,1992) basado en la física cuántica, donde la realidad en si 
misma no existe como tal sino que es algo subjetivo, el espacio lo conforma la visión que cada uno tiene del mismo y por tanto no existe una única visión sino que hay tantas como personas observan el territorio.

\section{SITUACIÓN ACTUAL}

\section{II.1. La sociedad líquida}

La sociedad ha cambiado, no podemos definir si nos encontramos ante un cambio de paradigma o un cambio de era pero desde luego que estamos ante un proceso de cambio muy profundo en un mundo global interconectado. La geografía se analiza desde un punto de vista industrial desde diversas perspectivas: desde la geografía cuantitativa basada en el neopositivismo y la objetivización que interpreta el mundo a través de los números a la geografía historicista que describe al mundo a partir de la historia, pasando por otra serie de análisis.

En los años setenta se produjo un cambio profundo en el desarrollo de la actividad económica con la aparición del toyotismo, un fenómeno como reacción al fordismo industrial (YASUHIRO, 1996). Mientras este último se basaba en la elaboración de procesos y cadenas de producción que generaban grandes volúmenes de productos y servicios a una sociedad más o menos homogénea, el toyotismo se basa en la filosofía «just in time» o «producción ajustada», donde se fabrica sólo la cantidad de productos y servicios que pueden venderse en el mercado adaptando estos a una segmentación del mismo.

La aparición de Internet hace que la conectividad sea cada vez mayor en todo el planeta haciendo que la globalización sea una realidad y las conversaciones derivadas de esa conectividad llegan a los mercados de la mano del Manifiesto Cluetrain y su máxima de «el mercado son conversaciones» (LEVINE y LOCKE, 2009).

El mundo ha cambiado y Bauman lo explica perfectamente con su concepto de sociedad líquida, una sociedad nueva, dinámica, flexible, rápida, cambiante, donde las personas y las organizaciones requieren de una gran capacidad de adaptación a los cambios, ya lo decía Darwin «la especie que sobrevive no es la más fuerte ni la más inteligente sino la que mejor se adapta al cambio». Frente a esto la sociedad antigüa basada en paradigmas clásicos, estática, inflexible, lenta, inmovilista, donde las personas y las organizaciones siguen usando viejas técnicas para resolver nuevos problemas.

\section{II.2. ¿Hacia una geografía liquida?}

La geografía es una de las ciencias a quien más afecta este cambio: los viejos mapas no sirven para manejarse en el nuevo mundo, los mapas de papel han 
sido substituidos por mapas online, las personas se comunican a través de las redes sociales, las empresas se vuelven abiertas y el trabajo es en red, la aparición de la nube como espacio virtual de trabajo, etc.

En este sentido nos encontramos antes una realidad del territorio con forma física, tangible, real y una traslación del territorio a Internet, donde la forma es digital, intangible, virtual. Pero lejos de pensar en dos geografías realmente hay una sola geografía con dos manifestaciones, hay una geografía liquida que se convierte en una herramienta de comunicación donde el mundo físico y el mundo digital se unen a través de la geolocalización, que se convierte así en una herramienta de comunicación. El hecho de que una persona realice un checking o diga donde está con su dispositivo móvil conectado por GPS supone que pasa de un espacio físico a un espacio digital a través de la nube, tal y como explica el manifiesto Solomo.

Pero la realidad científica es que se sigue analizando el territorio sólo desde un punto de vista, lo que hace que el análisis de la realidad quede afectada y de alguna forma «coja».

Es necesario entender cómo funciona esta sociedad líquida para poder analizar la realidad territorial de forma completa y por tanto ayudar en el estudio y transformación de la sociedad.

\section{II.3. La geografía 2.0.}

El término web 2.0. es un concepto desarrollado por Tim O'Reilly donde habla de una evolución de la web y un nuevo fenómeno de relación donde prima la bidireccionalidad.

El término fue acuñado en una conferencia en 2004 por Tim O'Reilly y John Battelle a partir de una comparativa entre los aspectos que cambiaban desde la Web 1.0 a la Web 2.0. Sintetizaron en 7 principios los aspectos que definían este nuevo concepto (O'REILLY, 2005): la Web como plataforma, el aprovechamiento de la inteligencia colectiva, los datos como el próximo Intel Inside, el fin del ciclo de versiones de software, modelos de programación ligeros, el software por encima del nivel de un único dispositivo y una experiencia de usuario más rica.

Fue el año 2006 cuando O 'Reilly, cerró una de las definiciones más aceptadas: «Web 2.0 es la revolución empresarial de la industria del software causada por su traslado hacia una Internet como plataforma e intentando entender las nuevas reglas de éxito de la misma. La principal de esas reglas es: construir 
aplicaciones que aprovechen el efecto red para que mejoren mientras más personas las usen (esto es lo que he llamado en otro lugar el «aprovechamiento de la inteligencia colectiva») (O'REILLY, 2006).

Siguiendo la reflexión de Edu Williams esta definición deja latentes tres conceptos clave sobre la Web 2.0 (WILlIAM y PÉREZ, 2008).

$\checkmark$ Es una revolución empresarial de una industria, la del software, y de ahí el prefijo Web. El paso del 1.0 al 2.0 es un movimiento dentro de dicha industria.

$\checkmark$ Los componentes de dicha industria (su cadena de valor) se trasladan a una plataforma común, Internet, donde poder desarrollar de manera óptima un sistema de redes.

$\checkmark$ El efecto de esas redes posibilitará el desarrollo de la industria basada en la inteligencia colectiva, donde son sus miembros los responsables de su construcción.

Siguiendo con esta reflexión la web 2.0., mas allá del concepto de herramientas sociales que se utiliza habitualmente, sería «el modelo que ha seguido la industria del software para adaptarse a la Economía del Conocimiento».

Por tanto la geografía 2.0. es un término que haría referencia a la adopción de estos supuestos y su aplicación a la geografía de este tercer milenio y está unida a la aparición de las herramientas de Social Media como nueva forma de comunicación.

La verdad es que el término web 2.0. está muy manido, según la Wikipedia «comprende aquellos sitios web que facilitan el compartir información, la interoperabilidad, el diseño centrado en el usuario y la colaboración en la World Wide Web».

Básicamente estamos hablando de la capacidad de generar una interacción entre el usuario y la web, frente a primigenia web en la que prácticamente no existía esa interacción. Hay más características que diferencian a la web 1.0. de la web 2.0. pero quizás la principal sea el paso de una comunicación unidireccional donde se emitía información en un solo sentido a una comunicación bidireccional donde la información se transmite de la web al usuario y del usuario a la web.

\section{LAS TECNOLOGÍAS DE LA INFORMACIÓN}

La tecnología como catalizador del cambio: en primer lugar, la aparición de los ordenadores y su incorporación al campo de la Geografía y, en segundo lugar, la aparición de Internet, tanto en el acceso a la información geográfica 
como en el acceso a herramientas para el tratamiento de esta información (RUIZ AlMAR, 2010).

El uso de las Tecnologías de la Información y la Comunicación (TICs) y de las Tecnologías de Información Geográfica (TIGs) son dos herramientas básicas para el desarrollo de los territories hoy en día y una no se entiende sin la otra. Las TIGs están vinculadas con el espacio dónde se desarrolla la actividad y la información que se genera en el mismo, mientras que las TICs se complementan al estar vinculadas con las personas que desarrollan la actividad y las conversaciones que se generan en el mismo.

Por tanto nos encontramos ante dos tipos de tecnologías: las TIG, propias del análisis del territorio y por tanto desarrolladas por los geógrafos y sobre todo vinculadas con los Sistemas de Información Geográfica en los que se utilizan los ordenadores y el análisis multivariable para realizar estudios y previsiones territoriales que de otra forma sería imposible realizar. Pero tal y como hemos explicado es necesario hablar de las TICs también y esta tecnología, mucho menos trabajada por los geógrafos, permite conectar directamente con el elemento humano, con las personas como los actores sociales de la comunicación en el territorio.

\section{III.1. La información geográfica}

\section{III.1.a. Big data y mapas}

Otro de los concpetos claves para entender la sociedad actual es el de Big Data. la importancia de los datos en un mundo hiperconectado. Se define como «todos aquellos conjuntos de datos cuyo tamaño supera la capacidad de búsqueda, captura, almacenamiento, gestión, análisis, transferencia, visualización o protección legal de las herramientas informáticas convencionales» (INVATTUR, 2015).

Esta definición está tomada de la publicación del Instituto Valenciano de Tecnologías Turísticas (INVATTUR, 2015) junto a Territorio Creativo: «Big Data: retos y oportunidades para el turismo», donde se habla de este concepto y se focaliza en el sector turístico.

El estudio va acompañado de una buena reflexión de José Luis Rodríguez y Mar Castaño: Por qué la industria del turismo invierte en Big Data: ¿supervivencia o cambio de modelo?, donde destaca esta frase que poco tiene que ver con la tecnología: «el gran reto de la transformación digital no son las herramientas, sino las personas y los principios que las mueven» (INVATTUR, 2015). 
Y es que detrás del Big Data hay cuatro dimensiones en torno a los datos: volumen, variedad, velocidad y valor. Pero ninguna de ellas tiene sentido si no le aportamos el valor humano; lo importante no son los datos en sí mismos, sino nuestra capacidad para interpretarlos y entenderlos.

En el caso del análisis de este concepto desde la geografía el foco está siempre en el apartado espacial, en la importancia del dónde y en este caso podría afirmar que gran parte de los datos tienen una componente espacial, una variable que nos habla de las coordenadas donde se generan esos datos.

Para analizar los datos desde una perspectiva espacial, desde los años noventa se utilizan los denominados Sistemas de Información Geográfica (SIG o GIS), unas herramientas cartográficas que trabajan con datos georreferenciados, en lo que algunos han denominado «mapamáticas» (unión de mapas y matemáticas), ya que se basan en el álgebra.

Estos SIG permiten visualizar numerosos datos atendiendo a las cuatro dimensiones del Big Data. Al fin y al cabo un mapa es una simplificación de la realidad y, por ello, nos permiten entender la realidad de un solo vistazo.

La clave de los SIG es que utilizan la geolocalización, es decir, las coordenadas geográficas, como nexo común entre el territorio y los datos que se van a analizar y mostrar.

Existen numerosas herramientas que permiten trabajar con datos y visualizarlos en un mapa. En función de quién genera esos datos, podemos hablar de tres tipos:

Privadas: Se trata de empresas privadas como Google que, a través de Mis Mapas de Google Maps o de Fusion Tables, permiten trabajar online con datos y mapas.

Públicas: Todas las Comunidades Autónomas y gran parte de los países europeos disponen de la Infraestructura de Datos Espaciales (IDE), una forma de homogeneizar los datos y ponerlos al servicio de los ciudadanos a través de geoportales como Terrasit.

Ciudadanas: La cartografía ciudadana se abre paso de la mano de Openstreetmap, la herramienta colaborativa donde los datos son públicos y abiertos para ser mejorados entre todos.

Estas herramientas ofrecen la posibilidad de crear mapas con datos geolocalizados, pero hay otras que se integran perfectamente con éstas y ofrecen posibilidades muy profesionales, como las españolas ArcGis Online de ESRI y 
la exitosa CartoDB, con un potencial enorme y sobre todo un prestigio merecido por las posibilidades que ofrecen.

En estos casos los datos geográficos pueden incorporarse a estos mapas de muy diversas formas: en diversos formatos como gpx (GPS profesionales), $\mathrm{kml} / \mathrm{kmz}$ (el formato de Google Earth) o shp (o Shapes, el formato básico de los SIG), a través de servidores web mediante WMS (Web Map Server) o georreferenciando sobre la misma web, mientras que las bases de datos pueden importarse a través del standard csv o xml.

La salida gráfica de estos datos también difiere de la forma tradicional y se puede dar de dos modos: offline, en forma de mapa para imprimir en papel o generando un pdf o similar; o bien en formato digital, donde se genera una URL o dirección web o un código HTML para embeberlo donde deseemos.

\section{III.1.b. La geolocalización}

Existe una confusión entre términos muy semejantes pero que son distintos: geolocalización es un término que se ha puesto de moda en Internet, utilizado muchas veces de forma similar a geoposicionamiento y georreferenciación; GPS como acrónimo de Global Positioning System (Sistema de Posicionamiento Global) y localización como un aspecto más genérico.

Lejos de intentar realizar un análisis semántico y conceptual de estos términos si que me resulta interesante acercarme al uso de estos términos tanto en Internet como en el lenguaje cotidiano, oficializado a través de la Real Academia de la Lengua Española, que es al fin y al cabo el organismo encargado de dotar de validez a los vocablos de nuestra lengua.

Geolocalización: la geolocalización es un concepto que hace referencia a la situación que ocupa un objeto en el espacio y que se mide en coordenadas de latitud (x), longitud (y) y altura (z). Aparece en la Wikipedia con la siguiente acepción: «La georreferenciación es un neologismo que refiere al posicionamiento con el que se define la localización de un objeto espacial (representado mediante punto, vector, área, volumen) en un sistema de coordenadas y datum determinado» (Wikipedia, 2015).

Pero en cambio no aparece en el Diccionario de la Real Academia de la Lengua ya que, como la propia Wikipedia, plantea, se trata de un «neologismo», es decir, de un nuevo concepto que aún no ha sido incorporado ni aceptado por la Comunidad Lingüística.

De hecho si analizamos Google Trends aparece como una tendencia a nivel mundial a partir del año 2011 pero aún no en España, por tanto, su importancia como para tener un uso considerable y medible es bastante reciente. 
GPS: en el Avance de la Vigésimo Tercera Edición del Diccionario de la Lengua Española de la Real Academia de la Lengua, sí aparece la palabras GPS hablando de un «Sistema que permite conocer la posición de un objeto móvil gracias a la recepción de señales emitidas por una red de satélites».

No deja de resultar curioso que se haya incorporado antes un elemento instrumental como es el GPS, al fin y al cabo un sistema entendido como herramienta, que un concepto teórico que sustenta el desarrollo de dicha herramienta.

Localización: lo más cercano que encontramos de forma «científica» es la definición de «Localizar» del Diccionario de la Real Academia de la Lengua, que en su tercera acepción habla de «Determinar o señalar el emplazamiento que debe tener alguien o algo» y quizás es lo que más se acerca a la definición de geolocalizar.

Por tanto en este artículo utilizamos el neologismo «geolocalización» como expresión popular más difundida de la localización de personas, objetos o cosas mediante el uso de un sistema GPS o similar.

\section{III.1.c. El geomarketing}

El geomarketing es una de las partes de análisis de la geografía humana desde hace décadas. Hay diversas definiciones de Geomarketing pero quizás una de las que más se acerque a la concepción del mismo sea la de CHASCO (2003): «Es un conjunto de técnicas que permiten analizar la realidad económico-social desde un punto de vista geográfico, a través de instrumentos cartográficos y herramientas de la estadística espacial».

Por tanto el geomarketing es un concepto que utiliza el análisis espacial como herramienta de marketing para la toma de decisiones y promoción de una organización, siendo una herramienta estratégica para el desarrollo de los negocios en un entorno determinado.

Una de las grandes revoluciones del geomarketing supuso la aparición y popularización de los Sistemas de Información geográfica a finales del siglo pasado, lo que permitió trabajar con grandes volúmenes de datos geolocalizados y asociados a información multivariable que ayuda a la toma de decisiones.

Otro de los grandes cambios ha venido dado por la aparición de Internet y la popularización de los mapas online, haciendo que el trabajo individual que se acumulaba, se gestionaba y se desarrollaba en los ordenadores personales pase a ser trabajado directamente en Internet a través del concepto de «nube» o «cloud». 
Con la aparición de la denominada web 2.0. y la popularización de los dispositivos móviles y los servicios de geolocalización (GPS) incorporados, se ha generado un cambio cualitativo y cuantitativo.

Este último cambio se ha producido con la aparición de los medios sociales en los que las personas generan información geolocalizada y la comparten, de forma que esos datos tienen un gran valor para comprender hábitos de consumo en el territorio.

\section{III.2. La información y la comunicación}

\section{III.2.a. Los medios sociales}

Por otra parte tenemos las redes sociales, que en realidad deberíamos traducir por «medios sociales», ya que es la correcta traducción de «Social Media», que en la Wikipedia se define como «plataformas de comunicación en linea donde el contenido es creado por los propios usuarios mediante el uso de las tecnologías de la Web 2.0, que facilitan la edición, la publicación y el intercambio de información» (Wikipedia, 2015).

La web 2.0. haría referencia a un entorno tecnológico y los medios sociales serían las plataformas de comunicación que permiten la comunicación de los usuarios con la web y entre ellos, pudiendo generar redes sociales como consecuencia de esas conexiones.

Las redes sociales no tienen nada que ver con la tecnología sino con el ser humano, que es sociable por naturaleza, lo que si que ha permitido Internet y la web 2.0. es que las personas puedan generar nuevas y amplias redes sociales mediante el uso de diversas plataformas que no son más que herramientas de comunicación.

Por tanto la clave de la unión entre el Social Media y la web 2.0. no está en las herramientas, en el medio, ya que éste puede cambiar y de hecho lo hace sino en el fin, que es la comunicación y la relación entre las personas y por eso se hace tanto hincapié en que al final lo que importa es la generación de contenidos de valor y de conversaciones.

\section{III.2.b. SoLoMo}

La geolocalización en Internet es una herramienta de comunicación entre la oferta y la demanda en un mundo que llamamos SoLoMo (Social, Local y Móvil): diariamente se genera una cantidad ingente de información (que no calidad), compartida a través de las redes sociales, con un componente local y a través de los móviles desde cualquier sitio. 
El acrónimo SOLOMO hace referencia al triángulo entre los conceptos de Social, Local y Móvil, tres aspectos en los que se basa gran parte de las estrategias de desarrollo de Internet hoy en día; y es atribuido a Matt Cutts, de Google.

En noviembre de 2011 se publica un manifiesto denominado «SOLOMO Manifesto» y bajo el subtítulo de «Just About Everything Marketers Need to Know About de Convergence of Social, Local, and Mobile (SoLoMo)», en el que se hace un exhaustivo repaso no sólo de las herramientas de geolocalización para el marketing en Internet sino el funcionamiento de las mismas (REED, 2011).

En este manifiesto se explica la importancia de los componentes de la geolocalización pero integrados en un análisis global donde la diferencia en el uso viene dado por la permanencia en el tiempo de las acciones.

La geolocalización es una herramienta de comunicación y por tanto hay tres partes:

$\checkmark$ El emisor, la oferta, es la persona o el negocio que se sitúa en el espacio, sea físico o digital

$\checkmark$ El receptor, la demanda, es la persona o el negocio que recibe una información localizada

$\checkmark$ El medio, la herramienta, es el móvil con un GPS incorporado y los programas que hacen compartir su localización

\section{LA GEOLOCALIZACIÓN SOCIAL}

\section{IV.1. Características}

Por tanto si unimos los conceptos que hemos citado previamente podríamos decir que la geolocalización y las redes sociales son dos caras de la misma moneda.

Cuando hablamos de geolocalización estamos diciendo que cualquier persona u objeto puede ser localizado en un punto en el espacio. Por tanto si unimos este concepto al de redes sociales estaremos hablando de la localización de las personas y negocios en el espacio que comparten en sus redes sociales para generar comunicación.

Este concepto hace referencia a las nuevas formas de relación social que surgen gracias a la geolocalización de los individuos con sus móviles y que pueden desarrollarse mediante diversas herramientas. 
Christian Palau habló de catorce principios de la Geolocalización Social (PALAU, 2010):

1. Los nichos sociales: el valor de la especialización y la creación de comunidad.

2. Solventar problemas en modo «just in time»: una necesidad, una solución a un clic.

3. Medir y volver a medir: el reto de ser usado por los usuarios, analizar todo dispositivos, uso, contenido, potenciales clientes.

4. Segmentar y segmentar: todos somos usuarios, pero cada uno hace un uso diferente.

5. A la caza del chollo: el regreso de los cupones. Los usuarios interactúan de forma diferente con el producto, quieren más información, que les sorprendan, sentir que controlan la situación.

6. Es mío: los dispositivos móviles se han convertido en una extensión de nuestro cuerpo y mente, son altamente personales, entrar en ellos es formar parte de la intimidad.

7. Nos gusta jugar: queremos jugar, no es una cosa de niños, incentivan la competitividad, permite compartir y retiene la atención.

8. Nuevos entornos: piensa globalmente y actúa localmente.

9. Creación de hábitos: todavía hay un número reducido de usuarios, por eso, poner fácil al usuario actividades habituales con nuevas posibilidades.

10.Seguridad: facilitar a los usuarios el control.

11.Del on al off y del off al on: con Internet en el móvil y la Geolocalización Social se difumina la frontera.

12. Maximizar la información: el Internet del futuro no se basa tanto en añadir nuevos contenidos como en saber maximizar el uso de la información existente.

13.Integración de plataformas: de contenidos, de canales, no podemos gestionar todos, al final se quedaran los mejores y los que no desaparecerán.

14.Propuesta de valor e integración en tiempo real. Los dispositivos adquirirán inteligencia y podrán sugerir acciones.

\section{IV.2. Objetivos}

A partir de aquí hay múltiples combinaciones de cosas que se pueden hacer con la geolocalización y las redes sociales y cómo todo dependerá de los objetivos que tengamos:

Localización: si lo que buscamos es que el negocio aparezca en los mapas online. 
Información: si lo que buscamos es informar sobre nuestro negocio o dónde estamos.

$\checkmark$ Tamaño: si lo que buscamos es tener seguidores para alcanzar una masa considerable.

$\checkmark$ Participación: si lo que buscamos es generar una comunidad de seguidores que participen en torno a un tema o a una marca.

$\checkmark$ Reputación: si lo que buscamos es gestionar la reputación online de nuestro negocio.

$\checkmark$ Tráfico: si lo que buscamos es generar tráfico a nuestra página web.

$\checkmark$ Posicionamiento: si lo que buscamos es aparecer en los primeros resultados de búsqueda en Google.

\section{IV.3. Perfiles}

La presencia en Internet se desarrolla de diversas formas que denominamos «perfiles». Una de las características comunes de las herramientas de geolocalización social es que podemos encontrar tres tipos de perfiles:

Personas: para poder usar una herramienta es necesario registrarse con un perfil personal en el que se pide normalmente un email, nombre de usuario y contraseña, aunque en ocasiones se solicite algún dato más. Se trata de la persona que va a gestionar el perfil o la cuenta corporativa, que posteriormente podrá tener otros administradores.

Empresas: son perfiles de página donde podemos encontrar diversas formas de crearlas, como marca, como organización, etc. pero se trata siempre de entidades. La persona o personas encargadas de administrar la página es el perfil personal anteriormente citado.

Lugares: puesto que la geolocalización es la base de este artículo y de las herramientas a utilizar una de las características diferenciadoras es que estas herramientas disponen de un perfil denominado lugar, cuya característica es que siempre está asociado a una dirección física.

Aunque puede resultar similar a la página en algunas herramientas se hace hincapié en éste aspecto que sobre todo afecta a la reputación online del sitio en concreto.

\section{IV.4. Herramientas}

Prácticamente todas las redes sociales tienen algún componente de geolocalización que con el creciente uso de los dispositivos móviles se va a convertir en imprescindible. 
Pero hay una serie de heramientas que funcionan de forma semejante y tienen características similares y son las que denomino herramientas de geolocalización social: Facebook Places, Google My Business, Foursquare y Yelp.

Se trata de aquellas herramientas que cumplen con las 14 características definidas por Christian Palau y otros criterios que las hacen comparables entre ellas y que, bajo mi punto de vista, serían los criterios por los que una herramienta se considera de geolocalización social:

1. Existencia de perfil de persona y página y/o lugar, aunque este último es el que criterio relevante.

2. Posibilidad de crear un lugar de forma social en Internet, es decir, sin necesidad de ser gestor ni administrador de ese negocio.

3. Posibilidad de gestionar la cuenta empresa reclamando el negocio.

4. Existencia de estadísticas internas de uso para analizar el comportamiento de los usuarios en función de su localización.

5. Localización de los negocios o recursos en una dirección concreta.

6. Posibilidad de generar comentarios sobre el lugar, opiniones que implican compartir una percepción subjetiva del lugar.

7. Posibilidad de generar puntuaciones sobre el lugar que implican compartir una percepción objetiva del lugar basado en un sistema de calificación por estrellas.

8. Posibilidad de realizar campañas de publicidad online basadas en la localización.

\section{IV.5. Funcionamiento}

El funcionamiento de la geolocalización y las redes sociales es sencillo:

$\checkmark$ El check-in: una persona está en un sitio y hace lo que se llama un check-in, decir dónde está con su móvil.

$\checkmark$ La localización: ese punto queda localizado en un mapa online.

$\checkmark$ La información: se incorpora información de ese lugar, puede ser objetiva-cuantitativa (puntuaciones) o cualitativa (opiniones), pero en cualquier caso afecta directamente a la reputación online del lugar.

$\checkmark$ La conversación: se comparte esa información en Redes Sociales a los seguidores de los usuarios, lo que genera un efecto de multiplicación y viralidad de la información.

$\checkmark$ La medición: estas acciones por parte de los usuarios dejan una huella digital que puede ser analizada y por tanto permite medir el resultado obtenido en función de los objetivos planteados. 
Por tanto a la hora de medir el uso de estas herramientas por parte de los usuarios debemos plantear una serie de métricas que, ponderadas por el criterio elegido, dará lugar a indicadores de geolocalización social o KPIs.

No es objeto de este artículo realizar un análisis ni una metodología de establecimiento de este sistema de indicadores pero si es importante indicar que existen las herramientas necesarias para hacerlo a través de los lugares analizados siempre que hayan sido reclamados para poder acceder a su gestión y monitorización.

\section{IV.6. Reputación online}

La reputación online es uno de los elementos que más se está analizando e implementando en el mundo del Social Media debido al gran impacto que tiene sobre las marcas y las personas. Con el desarrollo de los dispositivos móviles las personas y las marcas disponen de capacidad de movilidad, es decir, de desplazarse en el espacio y desde cada punto pueden generar información y compartirla en sus redes sociales, uniendo los tres elementos clave denominados SOLOMO (Social, Local y Móvil).

Pero los usuarios no sólo comparten su ubicación de forma objetiva (bajo unas coordenadas geográficas), sino que generan una información subjetiva en forma de opinión del espacio donde se encuentran y ahí es dónde hay una vinculación estrecha con la reputación online. Tal y como ya hemos indicado las herramientas de la geolocalización social afectan directamente a la reputación online de las marcas y los lugares, donde los comentarios de los usuarios pueden ejercer una influencia muy grande en dos sentidos:

a) Por una parte la opinión generada es compartida en diversos perfiles sociales, lo que magnifica su potencia y se viraliza de forma exponencial.

b) Por otra parte la opinión se genera en la propia página de la marca u organización, por lo que está visible para todos los usuarios de la misma. Los mapas como herramienta de comunicación.

\section{OTROS ASPECTOS DE LA GEOLOCALIZACIÓN SOCIAL}

\section{V.1. Los problemas de privacidad}

Uno de los elementos más controvertidos del uso de la geolocalización es el relativo a la privacidad. Ante el miedo inicial que genera esta cuestión hay que dejar claro como punto de partida que la geolocalización no es más que una herramienta por la que se puede conocer nuestra ubicación en el espacio. Por tanto debemos tratarla como tal, con la misma importancia que damos o dejamos de dar a otras herramientas y todo depende del uso que se le de. 
Es cierto que hace unos años los dispositivos móviles se vendían con la geolocalización activada, lo que deja un rastro de todas nuestras acciones en el espacio. Pero tras una serie de denuncias, la Unión Europea reaccionó rápidamente y el 16 de mayo de 2011 publicó el Dictamen 13/2011 sobre los servicios de geolocalización en los dispositivos móviles inteligentes, en el que se regulaba por primera vez vinculándolo con las leyes de protección de datos a favor del ciudadano.

También en el año 2011 INTECO publicó una interesante guía, yo diría que imprescindible, la Guía sobre seguridad y privacidad de las herramientas de geolocalización, con el objetivo de acercar al lector a este campo: en qué consiste la geolocalización, cuáles son sus elementos característicos, en qué tecnologías se apoya o cuáles son sus usos y aplicaciones principales.

Recientemente estamos viviendo un intenso debate sobre la polémica Ley de Geolocalización en México, declarada a principios de año como constitucional por la Suprema Corte de Justicia de la Nación y en la que hay un claro conflicto entre el poder del Estado y la libertad individual.

Por último, en todas las manifestaciones o confrontaciones sociales que estamos viviendo, el uso de la geolocalización es clave tanto para la organización entre las personas como para la localización de las mismas, convirtiéndose en un arma de doble filo para los usuarios. Precisamente Enrique DANS (2014) publicó un artículo denominado «Replanteando la red» en el que se comenta cómo está cambiando la configuración de los nodos de información.

En definitiva, el tema de la geolocalización y la privacidad es clave en estos tiempos pero también depende del punto de vista que se adopte en torno al mismo:

a) Desde el punto de vista de los usuarios, el control de la privacidad depende de la información que queramos dar y en este sentido podemos identificar dos aspectos:

El uso de la geolocalización en las redes sociales: cuando configuramos una cuenta, se nos suele preguntar sobre nuestro lugar de residencia y cuando interactuamos a través de las redes sociales la geolocalización aparece como opción, pero otras veces por defecto. En estos casos, si no queremos que se sepa dónde estamos o desde dónde generamos cierta información, podemos acudir a las mismas aplicaciones para desconectar la geolocalización y evitarlo.

El uso de la geolocalización en general: hay que tener en cuenta que cualquier acción que desarrollemos en Internet con la geolocalización del teléfono 
o del ordenador activada deja siempre un rastro y, por tanto, es susceptible de ser localizado. Por ejemplo hay una aplicación muy inquietante del mismo Google denominada «Historial de ubicaciones», que nos muestra dónde hemos estado en el último mes en función del uso de las aplicaciones, como puede ser la consulta del correo de Gmail.

b) Desde el punto de vista de los negocios, podemos utilizar estos datos siempre que sea de acuerdo con la ley y cumpliendo la protección de datos.

Existe un artículo muy interesante titulado «When enough is enough: location tracking, mosaic theory, and machine learning» y publicado en el New York University Journal of Law and Liberty donde se habla de geolocalización y privacidad pero desde una perspectiva distinta indicando que los peligros de la geolocalización no vienen tanto por los datos concretos que muestran la posición de una persona en un momento determinado, sino la suma de estos datos que, debidamente interpretados, pueden mostrar una tendencia que permita predecir esos movimientos y comportamientos y por tanto atenta directamente sobre la intimidad de las personas.

Por tanto la Teoría del Mosaico (ZIMMECK, 2014) se refiere a «un modelo empleado por los expertos en seguridad para extraer información sobre empresas, que se basa en la combinación de datos públicos con datos privados para extraer del mosaico resultante conclusiones sobre el comportamiento del sujeto analizado».

Como conclusion de este apartado y como aportación a la geografía podríamos indicar un elemento que pudiera parecer tecnológico como la geolocalización y la privacidad se convierte en un elemento sociólogico y, al centrar la atención en el espacio, eminentemente geográfico.

\section{V.2. La larga cola de la geolocalización}

Long tail o «larga cola» es el nombre coloquial para una característica de las distribuciones estadísticas (Zipf, Ley de potencias, distribuciones de Pareto y, en general, distribuciones de Lévy). En Internet se suele utilizar a la hora de hablar de las palabras clave que afectan al posicionamiento natural o SEO, pero en este caso se analizará desde otro punto de vista.

Básicamente se trata de mostrar que en los negocios hay unos pocos productos que abarcan la mayor parte del mercado y luego multitud de pequeños negocios que se reparten en el resto del mercado. De esta forma, podemos hablar de dos tipos de mercado muy distintos aunque complementarios:

El mercado de masas: centrado en el alto rendimiento de pocos productos y que, según algunos expertos, ya se está quedando atrás. 
El nicho de mercados: se basa en la suma o acumulación de todas las pequeñas ventas de muchos productos, que pueden igualar o superar al primero.

Uno de los planteamientos en torno a la geolocalización es cómo forma parte de la «larga cola», es decir, a menos que haya algo muy concreto para usarla, lo interesante es que aporta un valor añadido y diferencial que puede ayudar a cubrir partes de esa larga cola, de forma que mejore la competitividad del negocio.

Así, la suma de esas sencillas acciones y uso de herramientas de geolocalización puede ser tan importante como una gran acción centrada en un solo segmento del mercado.

\section{V.3. El geoposicionamiento emocional}

Existe una disciplina denominada neuromarketing, que proviene de la neurociencia que asegura que la mayor parte de nuestras decisiones son emocionales $\mathrm{y}$, por tanto, no se basan en criterios puramente racionales u objetivos. A partir de aquí se analizan las reacciones de las personas a determinados estímulos, de forma que conociéndolas podamos acercarnos más a sus necesidades.

El geoposicionamiento emocional es un concepto con el que trato de unir ambas cosas, para explicar el uso de la geolocalización teniendo en cuenta elementos emocionales y que puedan ser útiles para las personas, empresas y territorios.

Cuando hablamos de geoposicionamiento emocional, estamos diciendo que las personas son, en primer lugar, emocionales y eso lo transmiten en sus comunicaciones. Cada vez que alguien dice dónde está o hace un check-in, está generando una información emocional, positiva o negativa, y en menos ocasiones neutra.

También es verdad que de momento no podemos identificar esas emociones de forma automática, de hecho uno de los grandes problemas es la ironía del ser humano, que es difícilmente identificable por máquinas, ya que es un uso inteligente del lenguaje que además varía en cada lugar y en cada contexto, aunque se avanza hacia ahí.

Cada vez que una persona está en un sitio, emite una opinión. Si identificamos qué dice, podemos perseguir objetivos muy claros y definidos:

$\checkmark$ Generar con él una conversación.

$\checkmark$ Ofrecer atención al cliente.

$\checkmark$ Intentar atraerle a nuestro negocio. 
$\checkmark$ Obtener datos de su comportamiento.

$\checkmark$ Captarlo como seguidor.

$\checkmark$ Venderle productos o servicios directamente.

Aunque no existen herramientas específicas que nos permitan identificar ese «geoposicionamiento emocional», sí es verdad que podemos usar las habituales herramientas de geolocalización social para lograrlo.

Así pues este concepto trata de unir la geolocalización y las personas: lo primero como un elemento objetivo y racional, el decir dónde estamos; lo segundo como un elemento subjetivo y emocional, el decir qué sentimos; y sumando ambas cosas el «geoposicionamiento emocional», las emociones que generamos las personas en función de dónde nos encontramos.

Ésta es la vinculación directa con el territorio y el espacio local y en este sentido podemos encontrar puntos de encuentro con el sociólogo Diego Cerda, que ha realizado interesantes investigaciones en materia geosemántica, con afirmaciones que vinculan los mapas digitales con el espacio local «Los mapas digitales $(\ldots)$ son un medio de comunicación masiva, que permiten comunicar de manera efectiva una definición de un espacio geográfico que se identifica con un espacio vital para las comunidades locales» (CERDA, 2014)

De esta forma se produce una confrontación entre la geoestrategia tradicional y global frente a la microgeopolítica nueva a escala local y donde «los mapas oficiales se deslizan hacia un espacio del absurdo kafkiano de una soberanía sin significado en la realidad» (CERDA, 2014).

Por tanto geoposicionamiento emocional, geosemántica, mapas digitales, espacios y comunidades vitales locales aparecen como nuevos conceptos de la geografía donde de nuevo la geografía subjetiva aparece unida al mundo de Internet y su vinculación con el territorio.

\section{TENDENCIAS GEOESPACIALES}

El 12 de abril de 2012 el «Comité de Naciones Unidas de Expertos en Gestión Global de Información Geoespacial» publicó el documento «Tendencias futuras en la gestión de la información geoespacial», una reflexión de un número de expertos que a través de una amplia gama de aspectos de la comunidad geoespacial (expertos en la recogida de datos, los académicos y los principales usuarios de información geoespacial) fueron invitados a contribuir con sus puntos de vista sobre las tendencias emergentes en el mundo geoespacial.

En la reunión inaugural del Comité de Expertos en Gestión Global de Información Geoespacial (GGIM), celebrada en Corea en octubre de 2011, se decidió que había una necesidad de documentar los pensamientos de los líderes 
en el mundo geoespacial para el desarrollo futuro de esta mundo y en particular, el Comité se interesó en cómo estos cambios contribuirán a los programas estratégicos locales, nacionales y mundiales de crecimiento económico, la cohesión social y el bienestar, la sostenibilidad ambiental, la gestión de desastres, seguridad pública y el buen gobierno.

El entorno geoespacial va a ser un factor clave en el futuro de la sociedad: desde el desarrollo de una nueva comunicación hasta la legislación y la gobernanza territorial, pasando por nuevos desarrollos tecnológicos, nuevas posibilidades de negocio, la gestión de la formación o la prevención de riesgos naturales.

Pero a pesar de todos estos avances, la clave de estas tendencias va a estar en el factor humano, en la capacidad que tengamos de aunar los tres sectores:

a) Una iniciativa pública que debe enfrentarse a la tarea de legislar y poner en marcha nuevos desarrollos geoespaciales.

b) Una iniciativa privada que debe llevar estas tendencias al mundo empresarial para que sea un factor estratégico y práctico en el desarrollo de negocios.

c) Una iniciativa ciudadana, la posibilidad que tiene la ciudadanía de generar cartografía colaborativa que complemente a la generada desde la Administración y la empresa privada.

Así pues, de nuevo el ciudadano se configura como la clave de este triángulo y la llave que puede abrir las puertas a este nuevo futuro, más geoespacial que nunca.

\section{MÁS GEOGRAFÍA QUE NUNCA}

La reciente aparición de otros dispositivos denominados «wearables» como las gafas de realidad aumentada, los relojes inteligentes, las gafas de realidad virtual, etc hace que podamos preveer una importancia cada vez mayor de la geolocalización aunque no siempre en un primer plano. La venta por geolocalización o geocommerce aún no arroja resultados espectaculares pero en cambio el uso de la geolocalizacion en todos estos dispositivos es básico para cualquier acción aunque no sea aparentemente tan claro.

Por tanto si todos los dispositivos tienen un componente de geolocalización y lo llevan personas podríamos deducir que la geolocalización social tiene un largo recorrido. Cambiarán las herramientas y la forma de usarlas, cambiarán los perfiles de demanda y las pautas de consumo, pero el hecho de localizarnos en el espacio como personas o negocios seguirá estando ahí. 
La geografía tiene de nuevo la oportunidad de encontrar su lugar en el mundo analizando la nueva realidad desde la aparición de Internet y ejerciendo su función de unión y visión espacial imprescindible para el análisis de problemas complejos. Si la geografía física y la geografía humana fueron capaces de encontrarse en el análisis geográfico regional, la geografía tangible u offline y la geografía digital u online deberían unirse teniendo de forma equilibrada entre los dos aspectos que la hacen única: la capacidad de localizar en el territorio y la capacidad de aportar una vision humana al mismo, al fin y al cabo esa es la clave de la geolocalización social.

\section{BIBLIOGRAFÍA}

Bauman, Z. (2003): Modernidad líquida, Editorial Fondo de Cultura Económica, México DF

Bellovin, S. M.; Hutchins, R. M.; Jebara, T.; BoIRA, J.V. (1992): «El estudio del espacio subjetivo (Geografía de la Percepción y del Comportamiento: una contribución al estado de la cuestión», en Estudios Geográficos, nº 209, 573-592.

CAPEL, H. (1981): Filosofía y ciencia en la Geografía contemporánea. Una introducción a la Geografia. Barcelona: Barcanova.

CAPEL, H. (2010): «Geografía en red a comienzos del Tercer Milenio. Por una ciencia solidaria y en colaboración». Scripta Nova. Revista Electrónica de Geografía y Ciencias Sociales. [En línea]. Barcelona: Universidad de Barcelona, 1 de febrero de 2010, vol. XIV, $\mathrm{n}^{\mathrm{O}} 313$. http://www.ub.edu/geocrit/sn/sn-313.htm

CASTELLS, M. (2005): La era de la información, Tomo I, Economía, Sociedad y Cultura, Alianza Editorial, España

CERDA, D. (2014). «Microgeopolítica para la comunidad local. Geosemántica social y la sublimación del archivo KML de Google Earth». Escáner Cultural (Jul). http://revista.escaner.cl/node/7374

CHASCO, C. (2003): «El geomárketing y la distribución comercial». Investigación y marketing, 79, 6-13.

Christian, P. (2010): Programa ESIC \& ICEMD de Marketing Online en Sevilla http://www.slideshare.net/christianp/geolocalizacion-de-lo-global-a-lo-localmarzo-2011

DANS, E. (2014): «Replanteando la red»

http://www.enriquedans.com/2014/04/replanteando-la-red.html

DE SAINT-EXúPERY, A. (1998): El principito, Editora Latinoamericana, S.A. México.

GENís, R. (2014): Estrategias digitales para hacer crecer el negocio, Congreso de hoteleros españoles, 22-24 octubre 2014, Sta Cruz de Tenerife.

GIL, I. (2000): Sistemas y Tecnologías de la Información para la Gestión. Universidad Politécnica de Valencia.

Instituto Nacional de TeCnologías de la Comunicación (INTECO); Pérez SAN-JOSÉ. P. (dir.) (2011): Guía sobre seguridad y privacidad de las herramientas de geolocalización.

https://www.incibe.es/CERT/guias_estudios/guias/Guia_Geolocalizacion 
INVATTUR (VV.AA.) (2015): Big Data: retos y oportunidades para el turismo, Invattur.

http://www.slideshare.net/invattur/estudio-big-data-retos-y-oportunidades-parael-turismo.

LEVINE, R. \& LOCKE, CH. (2009): El manifiesto Cluetrain: el ocaso de la empresa, Deusto SA ediciones.

MirALBELL, O. (1999): «Visión estratégica de las organizaciones virtuales en el turismo: Aprovechamiento de las tecnologías de la comunicación y la información en la competitividad de las empresas turísticas». I Congreso «Turismo y Tecnologías de la Información y las Comunicaciones». TURITEC, 1999.

O'REILly, T. (2005): What is Web 2.0? (online) (September 2005).

http://oreilly.com/web2/archive/what-is-web-20.html?page=1 (accedido: 09/ 2014)

O'ReILly, T. (2006): Web 2.0 compact definition. Trying againg. (online) (December 2006).

http://radar.oreilly.com/archives/2006/12/web 20 compact.html (accessed: 03/09/2014)

PALAU, C. (2010): Geolocalización, de lo local a lo global, Programa ESIC \& ICEMD de Marketing Online en Sevilla.

http://www.slideshare.net/christianp/geolocalizacion-de-lo-global-a-lo-localmarzo-2011

REED, R. (2011): «SOLOMO Manifesto: Just About Everything Marketers Need to Know About de Convergence of Social, Local, and Mobile (SoLoMo)», Whitepaper.

RUIZ AlmaR, E. (2010): «Consideraciones acerca de la explosión geográfica: Geografía colaborativa e información geográfica voluntaria acreditada», GeoFocus (Artículos), $\mathrm{n}^{\circ} 10,280-298$

VV.AA. (2012): Future trends in geospatial information management: the five to ten year visión, UN Committee of Experts on Global Geospatial Information Management

Wikipedia, «Georreferenciación»

http://es.wikipedia.org/wiki/Georreferenciaci $\% \mathrm{C} 3 \% \mathrm{~B} 3 \mathrm{n}$

Wikipedia, «Social Media»

http://es.wikipedia.org/wiki/Medios sociales

WiLliAM, E y PÉREZ, E (2008): «La Web social como plataforma para desarrollar un ecosistema basado en el conocimiento». VII Congreso «Turismo y Tecnologías de la Información y las Comunicaciones». Turitec, 2008

YASUHIRO, M. (1996): El Just in time hoy en Toyota, Deusto SA ediciones

ZIMMECK, S. (2014): «When Enough is Enough: Location Tracking, Mosaic Theory, and Machine Learning», NYU Journal of Law \& Liberty, Vol. 8; U of Maryland Legal Studies Research Paper No. 2013-51, 555-628. 